\title{
Scaling and Intermittency in Animal Behavior
}

\author{
A. Harnos ${ }^{1}$, G. Horváth ${ }^{1}$, A. B. Lawrence ${ }^{2}$ and G. Vattay ${ }^{3}$ \\ ${ }^{1}$ University of Veterinary Science, H-1078 Budapest, István u. 2, Hungary \\ ${ }^{2}$ Genetics and Behavioural Sciences Department, The Scottish Agricultural College, \\ Bush Estate Penicuik, EH26 0QE, UK \\ ${ }^{3}$ Eötvös University, Department of Physics of Complex Systems, \\ H-1117 Budapest, Pázmány Péter sétány 1/A, Hungary
}

\begin{abstract}
Scale-invariant spatial or temporal patterns and Lévy flight motion have been observed in a large variety of biological systems. It has been argued that animals in general might perform Lévy flight motion with power law distribution of times between two changes of the direction of motion. Here we study the temporal behaviour of nesting gilts. The time spent by a gilt in a given form of activity has power law probability distribution without finite average. Further analysis reveals intermittent eruption of certain periodic behavioural sequences which are responsible for the scaling behaviour and indicates the existence of a critical state. We show that this behaviour is in close analogy with temporal sequences of velocity found in turbulent flows, where random and regular sequences alternate and form an intermittent sequence.
\end{abstract}

Scale-invariant spatial and temporal patterns have been observed in a large variety of biological systems [1]. It has been demonstrated that ants [2], Drosohyla [3] and the wandering albatross, Diomedea exulants [4] perform motion with power law distribution of times between two changes of the direction of motion. The power law distribution of times then leads to an anomalous Lévy type diffusion in space. In the last few years an increasing interest has been devoted to these superdiffusive processes in physics [5 [7]. and in econophysics [8 11]. Inspite of the extensive experimental studies the detailed mechanism responsible for the creation of the underlying power law distributions is not well understood. In this letter we demonstrate the first time that the power law and scaling observed in the behaviour of certain animals is related to intermittency, a phenomenon familiar from the theory of dynamical systems and turbulence.

It is well known that non-hyperbolic dynamical systems show superdiffusive behaviour. In dissipative systems it is caused by the trapping of trajectories in the neighborhood of marginally unstable periodic orbits [12]. The paradigmatic system showing such behaviour is Manneville's one dimensional map [13]

$$
x_{n+1}=x_{n}+c x_{n}^{z}(\bmod 1),
$$

where $z \geq 1$. Here the sequence $x_{n}$ spends long time trapped in the neighborhood of the marginally unstable periodic orbit (a fixed point) $x=0$. In analytic maps typically $z=2$. The invatiant density behaves as $\varrho(x) \sim x^{1-z}$ near the origin and it is not normalizable for $z \geq 2$. Accordingly the distribution of times spent by the sequence near the unstable periodic orbit has a power law tail.

Next we will show experimental evidence on the existence of unstable periodic patterns in animal behaviour.

Members of the species Sus scrofa invest considerable time and effort into building a nest before farrowing. Our aim was to investigate the temporal pattern of this highly motivated activity. Using time-lapse video we recorded the behaviour of 27 gilts and analyzed the last 24 hours preceding the farrowing. The experimental subjects were Large White X Landrace gilts (Cotswold Pig Development Company, Lincoln, UK). On day 109 of pregnancy they were moved to their individual farrowing accommodations. A behavioural collection program [15] was run to take data from the tapes. The behaviour of gilts was classified into eight mutually exclusive categories (see Table I). Further details of the experiment are published in Ref. [16].

As a first step we assigned a symbol $0,1, \ldots, 6$ to the 7 different types of behaviour listed on Table I. The records of the 27 gilts contain approximately 24.000 symbols. Then we computed the probabilities of the occurrences of symbolic sequences formed from the symbols. This has been done by evaluating the decimal value of the base seven number coded by the sequence. For example the code sequence 0156 is evaluated to be $\underline{0} * 1+\underline{1} * 7+\underline{5} * 7^{2}+\underline{6} * 7^{3}=2310$.

On the histograms of Fig. 1a, $1 \mathrm{~b}$ and $1 \mathrm{c}$ we show the probabilities of the length 3,4 and 5 sequences respectively. One can see that certain symbol sequences occur with high probability which does not decrease with increasing symbol length significantly, while the majority of symbols have relatively low probability and the occurrence of each particular symbol decreases with increasing length.

On the histogram of Fig. 2 we ordered the symbols of lengths $L=2,3$ and 4 according to decreasing probability. One can see that the tail of the histogram is exponential. An exponential histogram of probability ordered symbols is a property of random texts and reflects the fact that most of the behavioural patterns are generated by a Markovian process in a purely stochastic way. On the other hand, the most probable part cannot be considered as a result of 
random uncorrelated processes. On the histogram we can see that the probability of the most probable sequences does not decay significantly with increasing length and stays approximately constant for $L=2,3$ and 4 .

We have identified these most probable behavioural sequences. For gilts kept in pens the most likely sequence is the cyclic repetition of "nosing floor"-"alert"-"nosing floor"-... pattern. This sequence is the consequence of the nest building instinct which governs the behaviour of the animal most of the time. In crates however there is no possibility to try to build a nest due to the limited space and the absence of straw. Gilts become frustrated and the ideal "nosing floor"-" alert"-" nosing floor"-... sequence is occasionally interrupted by periods of rest. On Fig. 3 we show a typical record from a gilt kept in pen. We can see that the deterministic sequences of 1-6-1-... are dominant interrupted eventually by short random-like sequences of other symbols. This is very reminiscent of temporal sequences of velocity found in turbulent flows, where random and regular sequences alternate and form an intermittent sequence.

We can quantify this qualitative analogy by studying the probability of periodic sequences. On Figure $4 \mathrm{a}$ we show the probability to observe the periodic sequences "4-6-4-6-..." and "1-4-1-4-..." as a function of the length $L$ of the sequence. These are typical periodic symbolic sequences. To add a new symbol to an existing sequence in this case is approximately an uncorrelated, Markovian process. The probability decays exponentially with the length. On the other hand, the probability to observe the special periodic sequence "1-6-1-6-..." decays very slowly, according to the power law $1 / L^{2}$ for a wide range of $L$ values until an upper cutoff $L \approx 30$ is reached (Fig. 4b). This is in close analogy with intermittent flows, where the probability of regular velocity patterns decays according to a power law. The occurrence of the correlated sequences has a drastic effect on the probability distribution of the time the animal spends engaged in a given type of behaviour as we show next. On Fig. 5 we show the probability distribution of these times. The data can be fitted very accurately with the power law

$$
P(t)=C \frac{1}{\left(t+t_{0}\right)^{2}}
$$

where $t_{0}=21.3 \pm 0.6 \mathrm{sec}$. The exponent 2 of this power law is in accordance with the similar power law found for the probability of the "1-6-1-6-..." sequences. This function is valid between some lower $t_{l}$ and upper $t_{u}$ cutoff times. Based on the available data we have not reached these and we can say that $t_{l}$ is less than 30 seconds and $t_{u}$ is more than 2000 seconds. The distribution (2) is normalizable, however it has no first and higher moments. For example the average time spent in an activity

$$
\bar{t}=\int d t t P(t)
$$

does not exist. Taking into account that the validity of (2) is limited between lower and upper cutoffs the average time spent in an activity becomes $\bar{t} \sim t_{0} \ln \left(t_{u} / t_{l}\right)$, which is a very slowly growing function of $t_{u}$. However the variance of the time spent in an activity $(\overline{\Delta t})^{2} \sim t_{0} t_{u}$ and higher moments are very large making the behaviour of the animal very unpredictable.

It is important to note, that the observed power law distributions above are the same as those observable in the Manneville system 113,14,12 for $z=2$. This indicates that the sequence "1-6-1-6-..." marks a marginally unstable periodic orbit of the dynamics.

As a summary we demonstrated here, that the scaling behaviour in the animal behaviour found here is not related to environmental factors like in case of foraging animals, where the distribution of food might be distributed in a complex way forcing animals to follow Lévy flight patterns. The environment of gilts in pens and crates is almost absolutely unmotivating. The source of this complex behaviour can come only from the neural system forced by hormonal stimulus due to nesting instincts. This is the first carefully examined case, where complex scaling behaviour of animals is related to the self-organization and possibly to some unstable critical state of the nervous system. We hope, that further investigations of the behaviour of other types of animals subject to some internal hormonal pressure can be investigated and the existence of such a critical state can be established.

This work has been supported by the Hungarian Science Foundation OTKA (F17166/T17493/T25866) and the Hungarian Ministry of Education.

[1] West, B. J., Fractal Physiology and Chaos in Medicine (World Scientific, 1990).

[2] Schlesinger, M. F. in On Growth and Form (ed. Stanley, H. E. and Ostrowsky, N.) 283 (Nijhoff, Dordrecht, 1986). 
[3] Cole, B. J. Anim. Behav. 50, 1317-1324 (1995).

[4] Viswanathan, G. M. et al. Nature 381, 413-415 (1996).

[5] M. F. Shlesinger, G. M. Zaslavsky and J. Klafter, Nature (London) 363, 31 (1993)

[6] M. F. Shlesinger, G. M. Zaslavsky and U. Frisch (Editors), Lévy Flights and Related Topics in Physics, Springer Verlag Berlin (1996)

[7] J. Klafter, M. F. Shlesinger and G. Zumofen, Physics Today 49, 33 (1996)

[8] B. B. Mandelbrot, Fractals and scaling in finance: discontinuity, concentration, risk Springer, New York (1997)

[9] R. N. Mantegna, H. E. Stanley, Nature 376, 46-49 (1995); Nature 383, 587-588 (1996)

[10] J.-P. Bouchaud, D. Sornette, M. Potters, Option pricing in the presence of extreme fluctuations in Mathematics of Derivative Securities, ed. MA.H. Dempster, S. R. Pliska, Cambridge University Press (1997)

[11] S. Ghashghaie, W. Breymann, J. Peinke, P. Talkner, Y. Dodge, Nature 381, 767-770 (1996)

[12] T. Geisel and S. Thomae, Phys. Rev. Lett 52, 1936 (1984); T. Geisel, J. Nierwetberg, A. Zacherl, Phys. Rev. Lett. 54, $616(1985)$

[13] P. Manneville J. Phys. (Paris) 41, 1235 (1980)

[14] Ben-Mizrachi, A. Procaccia, I. Rosenberg, N. Schmidt, H. G. Schuster, Phys. Rev. A 31, 1830 (1985)

[15] Deag, M. J. Keytime: a Program System for Recording and Analizing Behavioural Data. Copyright John M. Deag 19871993.

[16] Horvath, G. and Lawrence, A. B. The effect of nest-building phase and the environment on the temporal pattern of gilts' neast-like activity to be published (1998) 


\begin{tabular}{|c|c|c|}
\hline Symbol & Behaviour & Definition \\
\hline 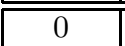 & Comfort & scratching \\
\hline 1 & Alert & $\begin{array}{l}\text { all other behaviours } \\
\text { not defined here }\end{array}$ \\
\hline 2 & Nosing environment & $\begin{array}{l}\text { exploratory or manipulative } \\
\text { behaviour directed at fixed } \\
\text { features of the environment } \\
\text { above floor level }\end{array}$ \\
\hline 4 & Rest & $\begin{array}{l}\text { lateral lying with the head } \\
\text { lowered and not 'nosing' }\end{array}$ \\
\hline 5 & Feeding or drinking & $\begin{array}{l}\text { head placed inside the } \\
\text { feeding or drinking trough }\end{array}$ \\
\hline 6 & Nosing floor & $\begin{array}{l}\text { exploratory or manipulative } \\
\text { behaviour directed at the } \\
\text { horizontal surface of the } \\
\text { floor or at the substrate thereon }\end{array}$ \\
\hline
\end{tabular}

TABLE I. The ethogram used to record the gilts' preparturient activity 

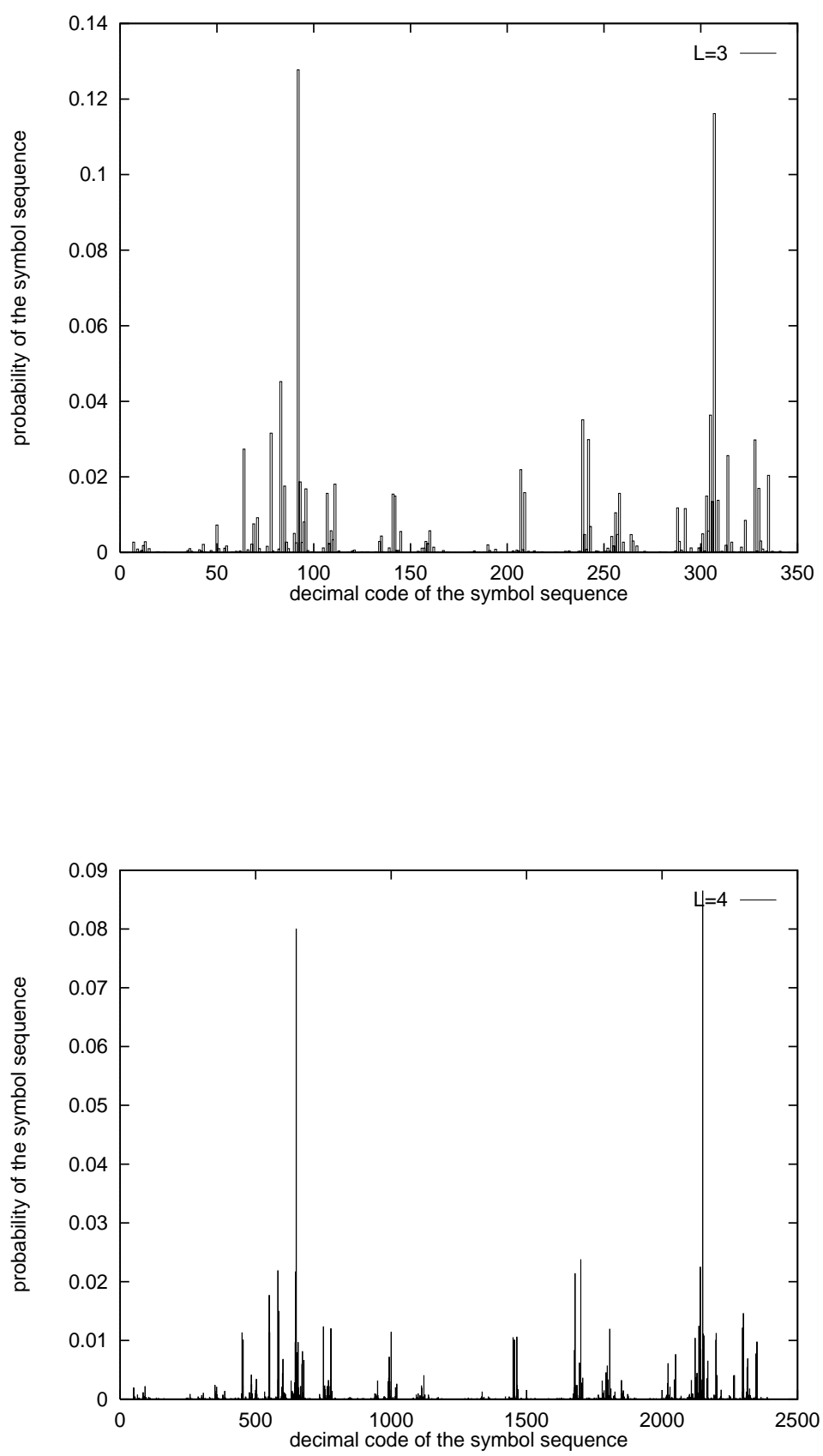


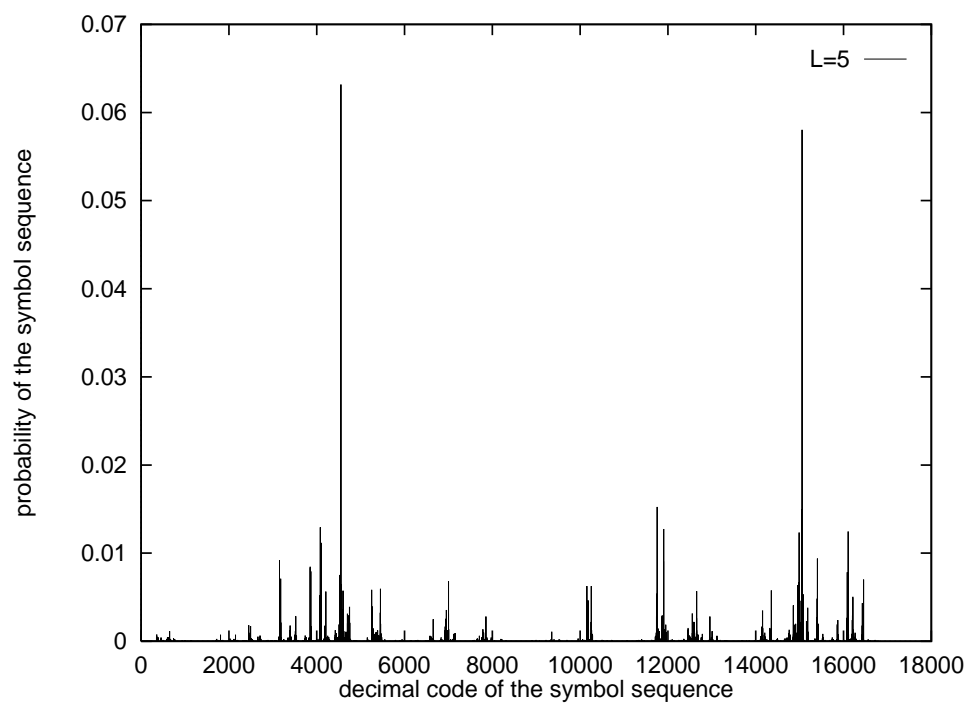

FIG. 1. Histogram of the probabilities of the symbolic sequences coding behavioural patterns. The video recorded animal activity has been translated into a code sequence of codes $0,1, \ldots, 6$ using the classification scheme of Table I. Then the sequential occurrence of length 1,2,3,4 and 5 code combinations of symbols had been analyzed. The symbol sequences are coded according to the value of the symbol sequence as a base 7 number. For example the length 4 code sequence 6510 is represented by the number $\underline{0} * 1+\underline{1} * 7+\underline{5} * 7^{2}+\underline{6} * 7^{3}=2310$ In this representation similar sequences get close to each other. This number is given on the horizontal axis and the corresponding probability is on the vertical axis and the length $L=3,4$ and 5 are shown on $\mathbf{a}, \mathbf{b}$ and $\mathbf{c}$ respectively. We can see that the probability of high probability sequences changes very slowly with $L$, while the rest of the probability is scattered among the increasing number of possible symbols. 


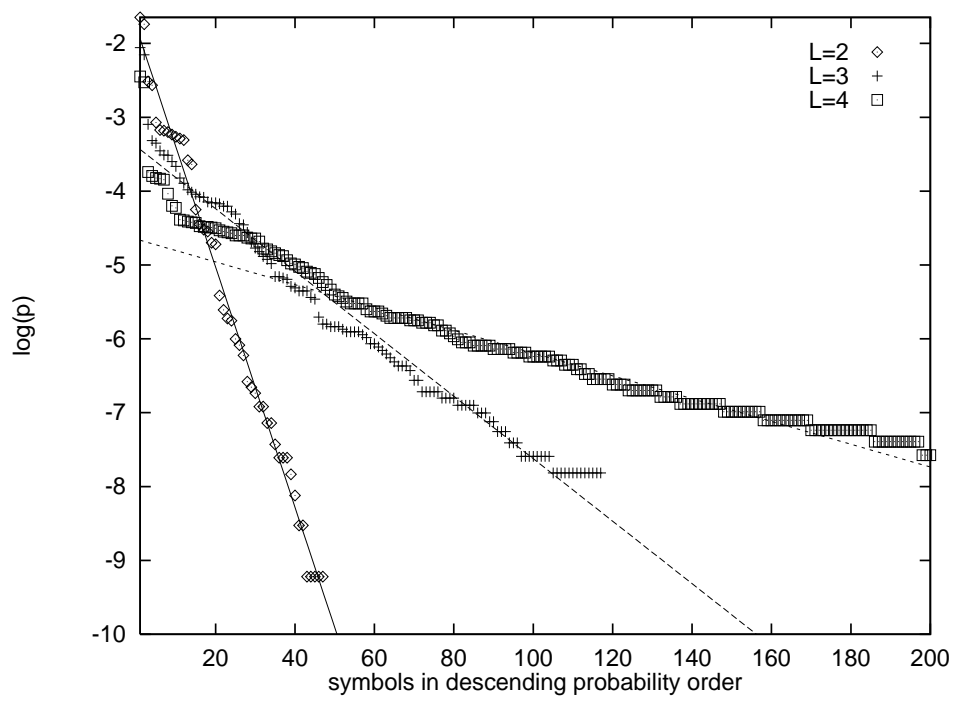

FIG. 2. The same histogram as on Fig. 1, however the symbols are now ordered according descending probability on the horizontal axis and on the vertical axis the logarithm of the symbol sequence probability is shown. The tail of the histogram can be fitted reasonably with an exponential. This is the typical behaviour of random symbol sequences generated by Markov processes. The head of the histogram remains almost in the same position indicating a highly correlated behaviour. These high probability sequences are codings of the periodic sequence "alert - nosing floor - alert -..." and its variants with "rest". These sequences are forced by the nest building instinct of the animals. 


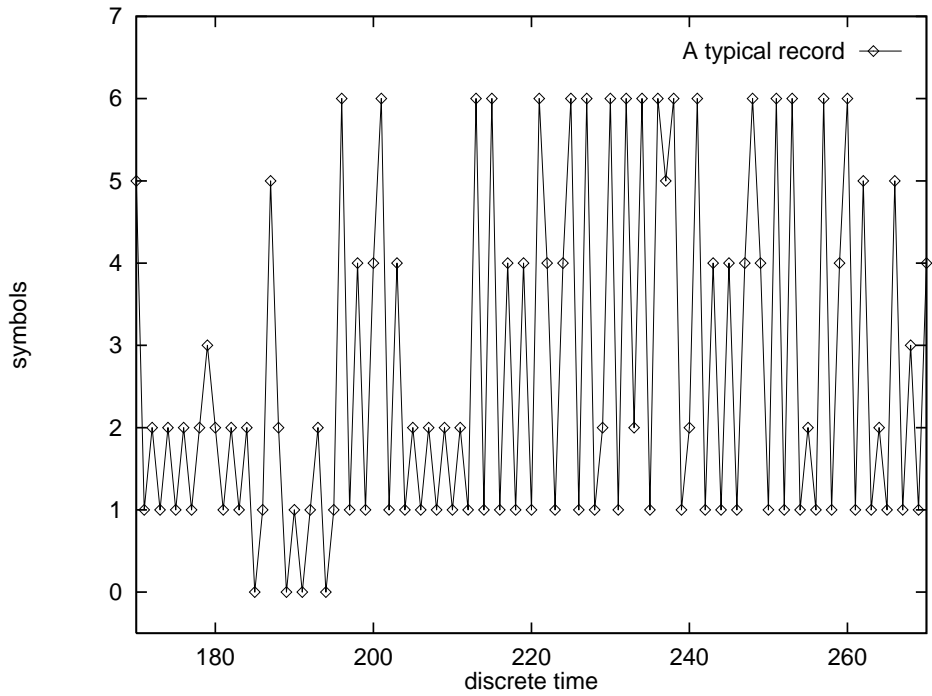

FIG. 3. A typical sequence from the time series. The horizontal axis is the (discrete) time while the symbols $0 \ldots 6$ are on the vertical axis. One can see clearly, that the "1-6-1-..." = "alert - nosing floor - alert -..." sequence dominates and it is interrupted eventually by other sequences. The random-like changes between the regular "1-6-1-6-..." parts are very similar to an intermittent time series typical in turbulence. 

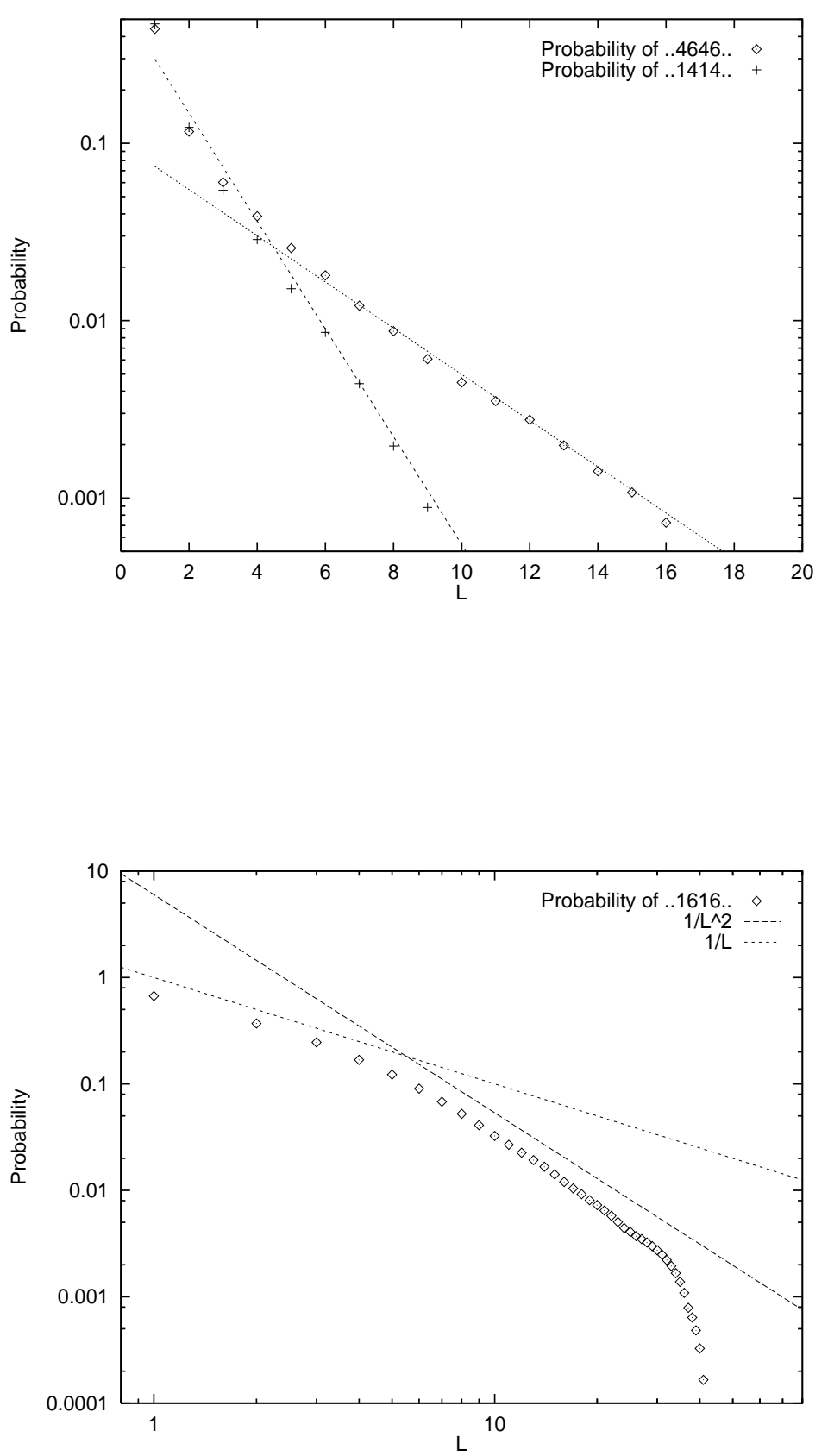

FIG. 4. Probability of the periodic sequences "1-4-1-4-..." and "4-6-4-6-..." a, and the probability of the periodic sequence "1-6-1-..." b, as a function of the length $L$ of the sequence. One can see that the probability of periodic sequences decays exponentially with the length except for the "alert - nosing floor - alert -..." sequence. The $1 / L^{2}$ power law decay is in close analogy with turbulent flows, where the probability of regular velocity patterns scales in a similar way. 


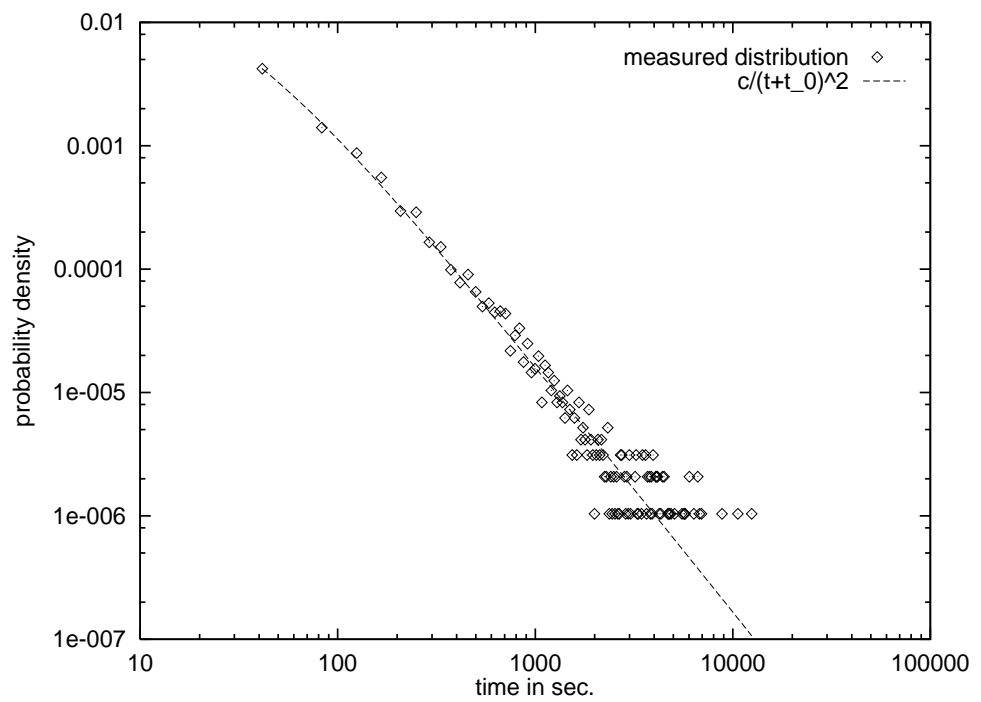

FIG. 5. Probability distribution of times spent in a given activity. The whole distribution can be fitted with the formula (1), where $t_{0}=21.3 \pm 0.6$ and $C=16.6 \pm 0.3$. The limits of validity of this formula are beyond the dataset available. The scaling behaviour of this distribution is a direct consequence of the neuro-hormonal behaviour of the animal. It is not a consequence of some scaling factors in the local environment of the animal. 